\title{
Turbulence in a free surface
}

\author{
W. I. Goldburg ${ }^{1}$, J. R. Cressman ${ }^{1}$, Z. Vörös ${ }^{1}$, B. Eckhardt ${ }^{2}$, and J. Schumacher ${ }^{2}$ \\ ${ }^{1}$ Department of Physics and Astronomy, University of Pittsburgh, Pittsburgh, PA 15260 \\ ${ }^{2}$ Fachbereich Physik, Philipps-Universität Marburg, D-35032 Marburg, Germany
}

(November 17, 2018)

\begin{abstract}
We report an experimental and numerical study of turbulent fluid motion in a free surface. The flow is realized experimentally on the surface of a tank filled with water stirred by a vertically oscillating grid positioned well below the surface. The effect of surface waves appears to be negligible so that the flow can numerically be realized with a flat surface and stress-free boundary conditions. The surface flow is unconventional in that it is not incompressible and neither energy nor enstrophy are conserved. Nevertheless, according to both experiment and numerical simulation, the second order structure function $S_{2}(R)$ scales essentially as for a three-dimensional system. However, the surface flow seems to be more intermittent.

PACS numbers: 47.27.Gs, 68.10.-m, 06.30.Gv
\end{abstract}

Statistically stationary turbulence is readily produced in a tank of water by vertically oscillating a grid below the water surface. The properties of this three-dimensional volume turbulence have been studied extensively [1]. Particles floating on the free surface are driven by the horizontal component of the turbulent flow below. While this is a classic problem, generally studied in an oceanographic context where the "particles" are micro organisms, such as phytoplankton [2], or Lagrangian drifters [3,4, controlled laboratory measurements of the relative velocity of floating particles do not appear to exist, despite a very large number of oscillating grid experiments which probe the fluid flow in the bulk and even near the surface [1]. Such surface flows also seem to have received little attention by those interested in the fundamental aspects of two or three dimensional turbulence [5]6]. Our experimental and theoretical results demonstrate that these two-dimensional surface flows have unusual properties that make them interesting in connection with scalar transport. The work opens the possibility of studying certain aspects of passive scalar dynamics in divergent flows beyond the ones used for the Kraichnan model which are Gaussian and white in time [7]. Their properties are also needed for a hydrodynamic modelling of the distribution of flotsam driven by underlying turbulence or chaos [8]. Our study is also related to recent work on quasi twodimensional turbulence, which is probed by tracking the motion of floating particles 91. The surface flows being comprised of particles constrained to the surface in both the experimental and numerical work, are qualitatively different from the motion of the driving fluid.

Although the fluid is incompressible, the inability of the floaters to enter the bulk flow assures that they will have a non-zero two-dimensional divergence. The compressibility of the floating particles causes them to clump, an affect seen in the numerical and experimental realizations. The main differences between their motion and that of the underlying fluid comes from the possibility that the floaters can exchange kinetic energy and enstrophy with the water molecules below. Hence, the energy and enstrophy of the floaters still would not be constants of the motion, even if the underlying fluid were invisid and undriven [10]. With the above conservation laws absent, one lacks the traditional dimensional arguments to estimate the scaling forms of the velocity and vorticity structure functions, $S_{2}(R)$ and $S_{\omega}(R)$. The structure functions are the main focus of our analysis and are defined as

$$
S_{2}(R)=\left\langle[(\mathbf{v}(\mathbf{x}+\mathbf{R})-\mathbf{v}(\mathbf{x})) \cdot \mathbf{R} / R]^{2}\right\rangle
$$

and

$$
S_{\omega}(R)=\left\langle[\omega(\mathbf{x}+\mathbf{R})-\omega(\mathbf{x})]^{2}\right\rangle,
$$

where $\mathbf{R}$ is the separation between two points and both $\mathbf{x}=\{x, y\}$ and $\mathbf{R}$ are in a horizontal plane. The vorticity, of magnitude $\omega(x, y)$, is perpendicular to that plane. A third quantity characterizing the surface flow is the dimensionless compressiblity coefficient $\mathcal{C}$, defined as

$$
\mathcal{C}=\left\langle(\nabla \cdot \mathbf{v})^{2}\right\rangle /\left\langle(\nabla \mathbf{v})^{2}\right\rangle
$$

which lies between 0 and 1 if the turbulence is isotropic [7]. As usual, $\langle\cdots\rangle$ denotes an ensemble average.

The measurements were made in a square plexiglass tank filled with water to a height that was varied. The bars of the grid were PVC and were square in cross section with dimensions of $1 \mathrm{~cm}$. The grid is also square and conforms closely with the square shape of the plexiglass box. To minimize flexing of the grid when it is oscillating, a number of the PVC bars are replaced by brass bars. For some of the measurements, a vertical post was placed at the center of the square grid to further suppress flexing, thereby reducing the amplitude of the surface waves. The apertures of the grid are also square, their dimensions being $L=3.66 \mathrm{~cm}$. The grid is supported by four vertical rods which run through pillow blocks, assuring its smooth vertical motion. All the parameters used in our set-up were typical of prior oscillating grid experiments [1], except that the lateral dimensions of the tank were somewhat larger. The grid is driven sinusoidally from above by a $1 / 4 \mathrm{hp}$ motor. The vertical amplitude of grid motion was $A=1.25 \mathrm{~cm}$. Whereas these parameters were varied 
by only a small amount, the distance $Z$ between the grid and the water surface $\left(\right.$ at $\left.z_{0}=0\right)$ was rather widely varied. Reducing $Z$ increases the Reynolds number of the turbulence on the surface and in the bulk close to the surface $\left(z_{0}>0\right)$. For all the measurements presented here, the oscillation frequency $f$ of the grid was $4.5 \mathrm{~Hz} \pm 0.3 \mathrm{~Hz}$.

The flow was measured using the technique of particle imaging velocimetry (PIV). There one illuminates the seed particles with a (horizontal) sheet of laser light and tracks their motion photographically. A CCD camera was centered at a distance that was $23 \mathrm{~cm}$ from the nearest wall. It was mounted above the tank where we could capture images of the surface particles or those in the interior. The particle illumination was furnished by a $\mathrm{Nd}$ Yag laser, with the beam spread into a horizontal sheet by a cylindrical lens. This sheet of light was typically 10 $\mathrm{cm}$ wide and $0.5 \mathrm{~cm}$ thick. The floaters were particles of various diameters ranging from 10 to $50 \mu \mathrm{m}$. The interior measurements were made using neutrally buoyant polystyrene spheres of diameter $10 \mu \mathrm{m}$. The images were acquired using a commercial particle imaging velocimetry apparatus [11 and the particle tracking was done using in-house software. To form a statistically reliable ensemble average, each measurement of $S_{2}(R)$ lasted several minutes and included $\sim 200$ image pairs. Each $S_{2}(R)$ was determined from roughly a million particle pairs.

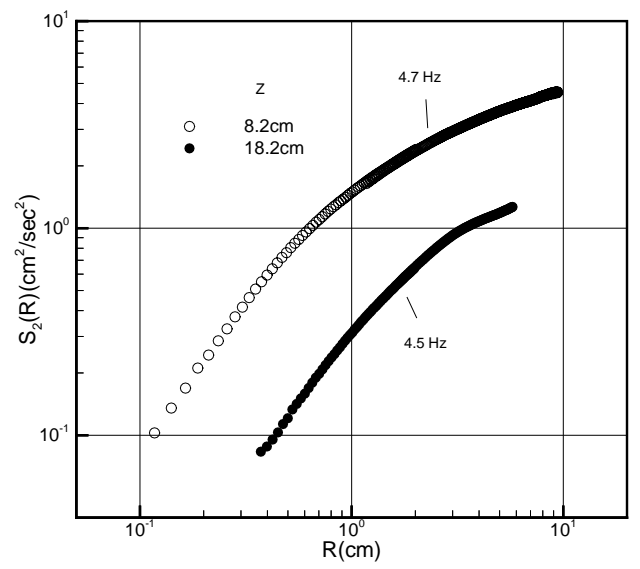

FIG. 1. Log-log plot of the second moment of the (longitudinal) velocity difference at the indicated distances $(Z)$ between the surface and the oscillating grid. The oscillating frequency of the grid and the surface-to-grid distances, $Z$, are indicated. Other parameters are $Z=8.2$ $\mathrm{cm}: R e_{\lambda}=80, l_{0}=3.6 \mathrm{~cm}, v_{r m s}=1.27 \mathrm{~cm} / \mathrm{s} ; Z=18.2 \mathrm{~cm}$ : $R e_{\lambda}=50, l_{0}=1.5 \mathrm{~cm}, v_{r m s}=0.7 \mathrm{~cm} / \mathrm{s}$. For both sets of measurements $\mathcal{C}=0.5 \pm 0.1$.

The surface and bulk turbulence are characterized by the outer scale of the turbulence $l_{0}$ and the Taylor microscale $\lambda$. The outer scale is defined as the integral of the normalized velocity autocorrelation function $C(R)=\langle v(x+R) \cdot v(x)\rangle / v_{r m s}^{2}$, where the brackets in- dicate an average over points $x, y$ and over many images. Here $v_{r m s}^{2} \equiv\left\langle\left(v_{i}(x, y)-\left\langle v_{i}(x, y)\right\rangle\right)^{2}\right\rangle$ where $i$ is one of the two arbitrarily chosen directions in the horizontal plane. Separate experiments established that the turbulence is almost isotropic in the horizontal plane (see below). The Taylor microscale is defined here as $\lambda=$ $\sqrt{\left(v_{r m s}^{2}\right) /\left\langle(d v(x) / d x)^{2}\right\rangle}$. The Taylor microscale Reynolds number is $R e_{\lambda}=v_{r m s} \lambda / \nu$, where $\nu$ is the kinematic viscosity (in water $\nu=0.01 \mathrm{~cm}^{2} / \mathrm{s}$ ). The surface motion is further characterized by the vertical component of the vorticity $\omega$ and by the dimensionless compressibility coefficient $\mathcal{C}$ [see Eq. (3)].

Figure 1 is a log-log plot of $S_{2}(R)$ in the surface measured for $Z=18.2 \mathrm{~cm}$ (closed circles) and for $Z=8.2 \mathrm{~cm}$ (open circles). The grid oscillation frequency for the two sets of measurements are indicated. The outer scale of the turbulence $l_{0}$ at $Z=18.2 \mathrm{~cm}$ and $8.2 \mathrm{~cm}$ are 1.5 and 3.6 $\mathrm{cm}$, respectively. The Taylor microscale Reynolds numbers $R e_{\lambda}$ are $80(Z=8.2 \mathrm{~cm})$ and $50(Z=18.2 \mathrm{~cm})$. Technical limitations blocked measurement of $S_{2}$ at $R$ less than $1 \mathrm{~mm}$. The measurements in Fig. 1 suggest that $S_{2}(R)$ is approaching its saturation value $2 v_{r m s}^{2}$ at large $R$. The more interesting case is $S_{2}$ at smaller $R$, where one finds that $S_{2}(R) \propto R^{\zeta}$, with $\zeta=1.6$ and 1.4 at $Z=$ 8.2 and $18.2 \mathrm{~cm}$, respectively. The dissipative range at even smaller $R$, where $S_{2}(R) \rightarrow R^{2}$, was not accessible in the laboratory experiment [5]. For these, and the other surface measurements, the dimensionless compressibility $\mathcal{C}$ defined above, was $0.5 \pm 0.1$. Reynolds numbers in the experiment and the simulation are too small to develop a significant interval with algebraic scaling in $S_{2}(R)$ between the dissipative, $S_{2}(R) \propto R^{2}$, and the saturation range $R \gg l_{0}$, where $S_{2}(R) \propto R^{0}$. Therefore the extended self similarity (ESS) representation is used [12] and a local scaling exponent $D_{2,3}(R)=$ $d \log _{e} S_{2}\left(\overrightarrow{R)} / d \log _{e} G_{3}(R)\right.$ for all $R$ in the interval $0.1 \mathrm{~cm}$ $<R<7 \mathrm{~cm}$ is found to give $0.70 \pm 0.10$ (see upper panel of Fig. 2). For the ESS plots, the third order structure function $G_{3}(R)=\left\langle|(\mathbf{v}(\mathbf{x}+\mathbf{R})-\mathbf{v}(\mathbf{x})) \cdot \mathbf{R} / R|^{3}\right\rangle$ is needed.

Since the surface is entirely driven by the gridgenerated turbulence in the water, it is important to measure $S_{2}(R)$ below the surface. We have made such measurements at distances $z_{0}$ in the range 0.5 to $2 \mathrm{~cm}$ and for various grid-to-surface distances $Z$. At $Z 9.6 \mathrm{~cm}$, $z_{0}=2.0 \mathrm{~cm}$, the second moment is very well fitted to a power law form in the interval $0.1 \mathrm{~cm}<R<4 \mathrm{~cm}$. In this interval $\zeta=0.9 \pm 0.2$, which is somewhat larger than the Kolmogorov value of $2 / 3$ [5]. Other relevant parameters for this bulk measurement are $R e_{\lambda}=100$ and $l_{0}=$ $3.1 \mathrm{~cm}$. The corresponding parameters measured on the surface are $R e_{\lambda}=40$ and $l_{0}=3.5 \mathrm{~cm}$. At all values of $R$, the bulk value of the second moment is roughly an order of magnitude larger than $S_{2}(R)$ measured at the surface. A dimensional argument suggests the crossover between bulk and surface behavior should take place at a depth $\delta z$ below the surface given by $\delta z \simeq \nu / \tilde{v}$ where 
$\tilde{v}$ is the bulk rms value of $v_{z}$. This gives $\delta z \simeq 0.1 \mathrm{~mm}$. The numerical studies 13 indicate that the prefactor in this dimensional estimate can be as large as 10 , making $\delta z \simeq 1 \mathrm{~mm}$, which is still too close to the surface for us to measure.

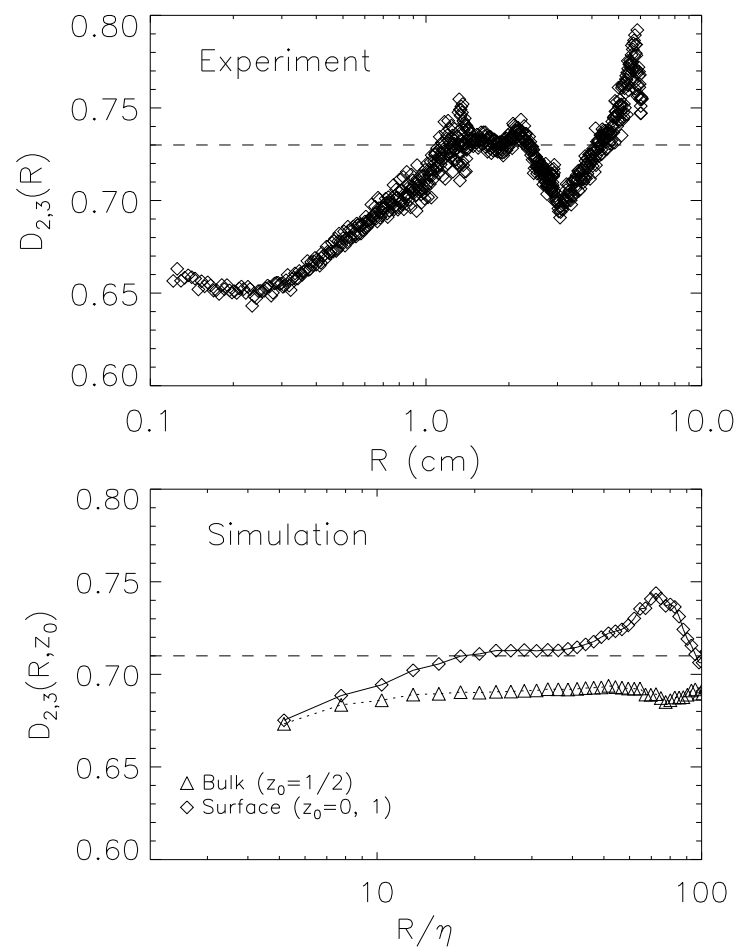

FIG. 2. Local scaling exponent, $D_{2,3}(R)$, of an extended self similarity plot of second order moment vs. absolute third order moment. Upper panel: The parameters for this experiment are the same as in Fig. 1 at $Z=18.2 \mathrm{~cm}$. Lower panel: Comparison of $D_{2,3}(R)$ in the bulk at $z_{0}=0.5$ (triangles) with that on the two surfaces at $z_{0}=0$ and 1 (diamonds). Data were taken from a numerical simulation at $R e_{\lambda} \simeq 100$. Dashed lines in both panels are drawn at $D_{2,3}(R)=0.73$ and 0.71 , respectively.

We consider the possibility that the surface velocity fluctuations originate from gravity and capillary waves generated by the $4.4 \mathrm{~Hz}$ oscillation frequency of the grid. Even though the amplitude of the surface waves was rather small $(A \simeq 0.5 \mathrm{~mm}$ ) it is comparable to the thickness of the boundary layer near the surface. The appropriate wave turbulence theory treats the nonlinear coupling of surface modes $\Omega(k)$ of different wavenumbers $k$ 14. This theory is of Hamiltonian form, which requires that the (2D) velocity field in the theory is obtained from a velocity potential, so that the vorticity of the surface particles is zero. Measurements of the (vertically-directed) vorticity of the surface particles show that it is quite large. Figure 4 is a log-log plot of the second moment of the surface vorticity fluctuations, $S_{\omega}(R)$, for a grid-to-surface distance $Z=8.5 \mathrm{~cm}$. This function saturates at a rather small value of $R$ and approaches the limiting value $2\left\langle\omega^{2}\right\rangle$. A dimensionless measure $(Q)$ of the surface vorticity is
$S_{\omega}(R) R^{2} / S_{2}(R)$. At $R$ equal to the outer scale of the turbulence, $l_{0} \simeq 3 \mathrm{~cm}, Q \simeq 1$, which suggests that it is probably too large to satisfy the zero-vorticity requirement of wave turbulence theory, even though this theory successfully accounts for the motion of particles floating on a container of vertically oscillating fluid where capillary waves are excited [15,16.

The experimental observations are, however, in good agreement with a model for turbulence in a flat surface bounding a three-dimensional volume with fully developed turbulence governed by the incompressible NavierStokes equation 13. This fluid is in a computational box with periodic boundary conditions applied to the lateral edges but with the fluid particles on the upper and lower surfaces obeying free-slip boundary conditions, with $v_{z}=\partial_{z} v_{x}=\partial_{z} v_{y}=0$. The equations were solved by means of a pseudospectral method with resolution $N_{x} \times N_{y} \times N_{z}$ of $256 \times 256 \times 65$. A statistically steady state of the turbulent flow was achieved by forcing in a wavenumber range comparable to the inverse of the box height (for more details see [17]). Here $R e_{\lambda} \simeq 100$.

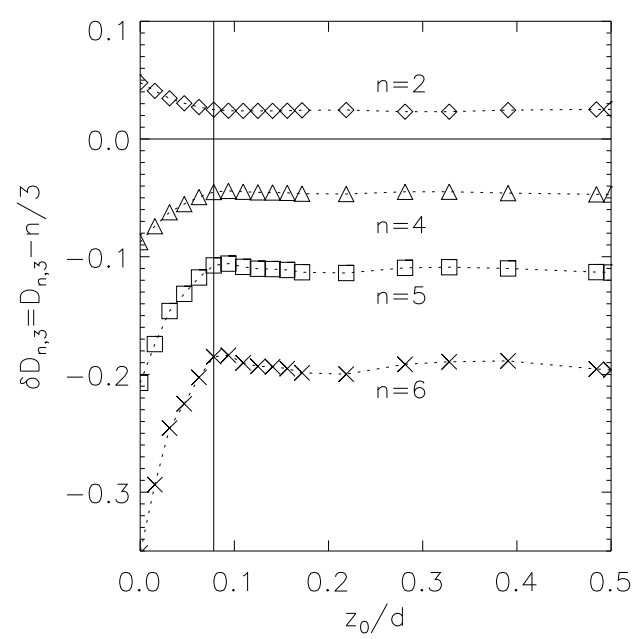

FIG. 3. Deviations of the local extended self-similarity (ESS) slope from the classical Kolmogorov scaling for different heights $z_{0}$ of the averaging plane and for different orders $n=2$ to $n=6$. All data points are calculated from $2 \times 114$ statistically independent samples at $R e_{\lambda} \simeq$ 100 and by averaging $D_{n, 3}\left(R, z_{0}\right)$ for scales $R$ between $18 \eta$ and $41 \eta$.

The local ESS scaling exponent $D_{2,3}(R)$ lies in the range between 0.67 and 0.74 in the interval $5<R / \eta<100$ with the Kolmogorov length $\eta=\left(\nu^{3} / \epsilon\right)^{1 / 4}$ (lower panel of Fig. 2) . This result is in good agreement with the experimental data also shown in Fig. 2 (upper panel). The simulation results show a plateau between $18 \eta$ and $41 \eta$ for the local scaling exponents in both cases, at the surface and in the bulk. Intermittency corrections in the 
surface seem to be stronger than in the bulk. In Fig. 3, a systematic investigation of these deviations from the classical Kolmogorov scaling with respect to $z_{0}$, the vertical position of the horizontal plane of averaging is presented. Our results indicate increasing deviations toward the free surface. The two-dimensional surface compressibility $\mathcal{C}$ in the calculation is 0.5 , in excellent agreement to the laboratory-measured value of $0.5 \pm 0.1$. This value of $\mathcal{C}$ is expected for isotropic turbulence.

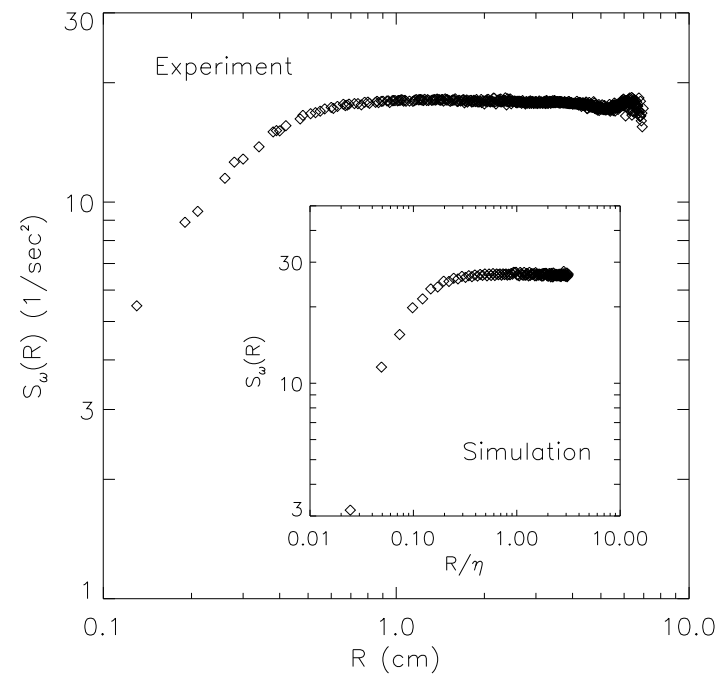

FIG. 4. Second moment of vorticity differences for the floating particles, at $Z=9.6 \mathrm{~cm}$. Here $f=4.7 \mathrm{~Hz}, R e_{\lambda}=35$, $l_{0}=1.7 \mathrm{~cm}$, and $v_{r m s}=0.67 \mathrm{~cm} / \mathrm{s}$. Again $\mathcal{C} \simeq 0.5$. Inset: Model calculation of $S_{\omega}(R)$ at the free-slip surfaces, with $R e_{\lambda} \simeq 100$. Here $\mathcal{C}=0.5$.

The above model was also applied to the calculation of $S_{\omega}(R)$, and the results are presented in the inset of Fig. 4. Both the calculated and measured $S_{\omega}(R)$ are qualitatively similar and show that this function rises rapidly with increasing $R$ in these log-log plots and reaches its saturation value, $2\left\langle\omega^{2}\right\rangle$ at a rather small value of $R$.

In summary, we have described a laboratory experiment and a model calculation for particles that float on a sea of three-dimensional turbulence. Even though the surface particles move in a plane, there appears to be no basis for describing the floating particle system in terms of the laws of two-dimensional turbulence or of turbulent surface waves. Important characteristics of the floating particle system are its large compressibility, large vorticity and the absence of conservation laws for energy and enstrophy. The measurements and calculations presented here indicate the existence of an inertial range where $S_{2}(R)$ scales as $R^{\zeta}$, with $\zeta$ quite close to $2 / 3$, as in $3 \mathrm{D}$ flows and in the inverse cascade of $2 \mathrm{D}$ turbulence.

In this work we have profited from interactions with $\mathrm{P}$. Alstrøm, S. Banerjee, D. Lohse, C. Cheung, G. Falkovich,
V. Horváth, M. Rivera, E. Schröder, X. L. Wu, and M. Vergassola. The particle tracking software of M. Rivera was very important to the success of this experiment. Derek Hohman provided valuable assistance with the experiment. The work has been strongly influenced by the attendance of two of us (W.I.G., B.E.) at the Institute for Theoretical Physics at Santa Barbara. Research support from NASA, the National Science Foundation, and the European Community is gratefully acknowledged. We thank the John von Neumann-Institut für Computing at the Forschungszentrum Jülich for support and computing time on a Cray T-90 for the numerical simulations.

[1] N. Matsunaga et al., Fluid Dyn. Res. 25, 147 (1999). This reference contains a complete bibliography of prior experiments on oscillating grid turbulence. For related studies of particle motion near the surface in channel flow, see Y. Pan and S. Banerjee Phys. Fluids 7, 1649 (1995) and S. Kumar, R. Gupta, and S. Banerjee, Phys. Fluids 10, 437 (1991).

[2] J. F. R. Gower, K. L. Denman, and R. J. Hoyler, Nature 294, 673 (1980).

[3] H. Stommel, J. Marine Research 8, 199 (1949).

[4] R. E. Davis, Annu. Rev. Fluid Mech. 23, 43 (1991).

[5] U. Frisch, Turbulence, (Cambridge University Press, Cambridge, 1995).

[6] M. Lesieur, Turbulence in Fluids, (Kluwer Academic Publishers, Dordrecht, 1990), 2nd edition.

[7] K. Gawędzki and M. Vergassola, Physica D 138, 63 (2000).

[8] T. Antonsen et al., Phys. Rev. Lett. 75, 3438 (1995); A. Namenson et al., Phys. Fluids 8, 2426 (1996); J. C. Sommerer, Phys. Fluids 8, 2441 (1996).

[9] M.-C. Jullien, J. Paret, and P. Tabeling, Phys. Rev. Lett. 82, 2872 (1999).

[10] R. H. Kraichnan, Phys. Fluids 10, 1417 (1967).

[11] TSI Inc., Fluid Mechanics Instrument Division, 500 Cardigan Rd., St. Paul MN 55126, E-mail: fluid@tsi.com.

[12] R. Benzi, S. Ciliberto, R. Trippicone, C. Baudet, F. Massaioli, and S. Succi, Phys. Rev. E 48, R29 (1993).

[13] B. Eckhardt and J. Schumacher, Turbulence and passive scalartransport in a free-slip surface, submitted (2000).

[14] V. E. Zakharov, V. S. L'vov, and G. Falkovich, Kolmogorov Spectra of Turbulence I, Wave Turbulence (Springer-Verlag, Berlin, 1992) and references therein; P. Alstrøm "Surface Waves - Patterns, Instabilities, and Turbulence" (Niels Bohr Institute, Copenhagen, Denmark), 1999 (unpublished).

[15] E. Schröder et al., Phys. Rev. Lett. 76, 4717 (1996).

[16] E. Schröder and P. Alstrøm, Phys. Rev. E 57, 7329 (1998).

[17] J. Schumacher and B. Eckhardt, Europhys. Lett. 52, 627 (2000). 